
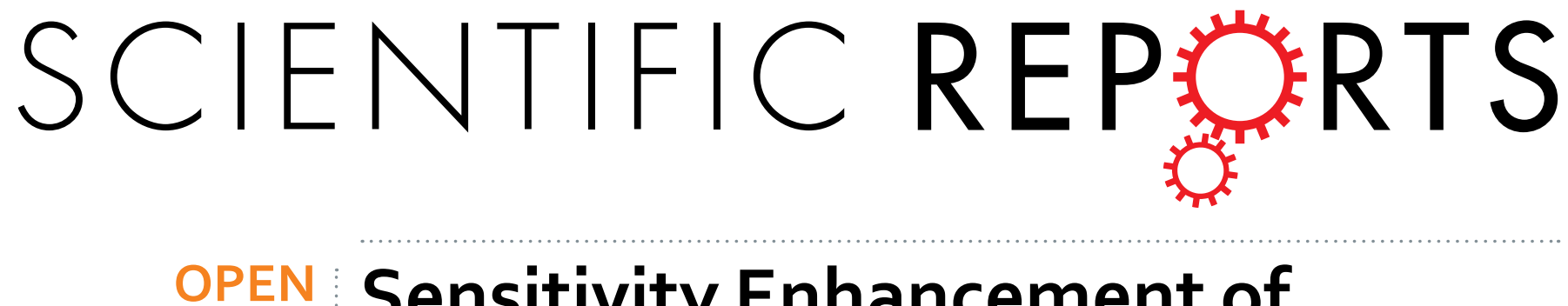

\title{
Sensitivity Enhancement of
} Transition Metal Dichalcogenides/ Silicon Nanostructure-based

Received: 15 March 2016

Accepted: 01 June 2016

Published: 16 June 2016
Surface Plasmon Resonance Biosensor

\begin{abstract}
Oingling Ouyang ${ }^{1,2, *}$, Shuwen Zeng ${ }^{1,2, *}{ }^{,}$, Li Jiang ${ }^{2,3}$, Liying Hong ${ }^{1}$, Gaixia Xu ${ }^{2,4}$, Xuan-Quyen Dinh ${ }^{2}$, Jun Qian ${ }^{3}$, Sailing He ${ }^{3}$, Junle $\mathbf{Q u}^{4}$, Philippe Coquet ${ }^{2,5}$ \& Ken-Tye Yong ${ }^{1,2}$
\end{abstract}

In this work, we designed a sensitivity-enhanced surface plasmon resonance biosensor structure based on silicon nanosheet and two-dimensional transition metal dichalcogenides. This configuration contains six components: SF10 triangular prism, gold thin film, silicon nanosheet, two-dimensional $\mathrm{MoS}_{2} / \mathrm{MoSe}_{2} /$ $\mathrm{WS}_{2} / \mathrm{WSe}_{2}$ (defined as $\mathrm{MX}_{2}$ ) layers, biomolecular analyte layer and sensing medium. The minimum reflectivity, sensitivity as well as the Full Width at Half Maximum of SPR curve are systematically examined by using Fresnel equations and the transfer matrix method in the visible and near infrared wavelength range $(600 \mathrm{~nm}$ to $1024 \mathrm{~nm})$. The variation of the minimum reflectivity and the change in resonance angle as the function of the number of $\mathrm{MX}_{2}$ layers are presented respectively. The results show that silicon nanosheet and $\mathrm{MX}_{2}$ layers can be served as effective light absorption medium. Under resonance conditions, the electrons in these additional dielectric layers can be transferred to the surface of gold thin film. All silicon- $\mathrm{MX}_{2}$ enhanced sensing models show much better performance than that of the conventional sensing scheme where pure Au thin film is used, the highest sensitivity can be achieved by employing $600 \mathrm{~nm}$ excitation light wavelength with $35 \mathrm{~nm}$ gold thin film and $7 \mathrm{~nm}$ thickness silicon nanosheet coated with monolayer WS .

Surface plasmon resonance (SPR)-based biosensors have attracted great attention as one of the leading optical sensing technologies during the last two decades due to their unique advantages such as real-time and label-free detection $^{1-3}$. They played an important role in monitoring various biomolecular interactions like protein bindings and DNA hybridization ${ }^{4,5}$. Further applications such as pharmacology and early disease diagnostics are promising if the SPR sensitivities have a drastic improvement ${ }^{6}$. The first observation of the SPR dated back to 1902, however, the complete explanations on this phenomenon were not provided by the scientific community until 1968 when Kretschmann, Raether and Otto proposed effective excitation configurations and abundant theoretical demonstration ${ }^{7,8}$. The surface plasmons (SPs) can be considered as electron density waves that propagate at the interface between metal and dielectric. In order to excite the surface plasmon waves (SPW), the incident photons or electrons should oscillate with the free electrons on the metal surface to form the resonances. Only $p$-polarized light (TM waves) contributes to the excitation of the SPs, while $s$-polarized light (TE waves) could act as the reference signals. When the horizontal component of the incident wave vector $k_{x}$ (i.e., the evanescent wave vector)

${ }^{1}$ School of Electrical and Electronic Engineering, Nanyang Technological University, Singapore, 639798. ${ }^{2} \mathrm{CINTRA}$ CNRS/NTU/THALES, UMI 3288, Research Techno Plaza, 50 Nanyang Drive, Border X Block, Singapore, 637553. ${ }^{3}$ State Key Laboratory of Modern Optical Instrumentation, Centre for Optical and Electromagnetics Research, Zhejiang University, Hangzhou 310058, China. ${ }^{4}$ Key Laboratory of Optoelectronics Devices and Systems of Ministry of Education/Guangdong Province, College of Optoelectronic Engineering, Shenzhen University, Shenzhen, P. R. China. ${ }^{5}$ Institut d'Electronique, de Microélectronique et de Nanotechnologie (IEMN), CNRS UMR 8520 - Université de Lille 1, 59650 Villeneuve d'Ascq, France. *These authors contributed equally to this work. Correspondence and requests for materials should be addressed to S.Z. (email: swzeng@ntu.edu.sg) or J.Q. (email: jlqu@szu.edu.cn) or K.-T.Y. (email: ktyong@ntu.edu.sg) 
matches with the surface plasmon wave vector $k_{s p}$, the surface plasmon resonance phenomenon occurs, as shown in equation (1),

$$
k_{x}=k_{0} n_{\text {prism }} \sin \theta_{\text {inc }}=k_{s p}
$$

where $k_{0}$ represents the incident wave vector in free space and $\theta_{\text {inc }}$ denotes the incident angle. Here, the incident angle is known as the resonance angle (or SPR angle). When the excitation light wavelength was fixed, one can obtain a SPR curve with a dip by scanning the incident angle and monitoring the reflectivity. The minimum of the reflectivity (nearly zero) corresponded to the reflected intensity at the resonance angle. When the SPW was excited, part of the incident optical energy was converted into the SPW resonance energy, leading to the drastic decrease in the reflected intensity. Ideally, all incident energy should be absorbed to support the resonant oscillations and result in a strong evanescent field. For the SPR biosensor, the SPR angle serves as an important output signal for the angular interrogation system. Since the refractive index changes of the sensing layer that are induced by the adsorption of biomolecules on the sensing surface would lead to a redistribution of SPR electromagnetic field, a significant SPR angle shift could be obtained and collected through an optical detector. This unique characteristic enables SPR biosensors to show excellent performances for real-time and label-free detections. However, the sensitivity is known to be limited especially when the weight of the biomolecules analyte is less than $500 \mathrm{Da}^{9}$. Various methods are provided to enhance the sensitivity: Silver thin film as SPR sensing substrate was demonstrated to have better performance than that with gold in sensitivity, however the weak chemical stability of silver impedes its further development ${ }^{6}$; In addition, the coating of an additional dielectric nanolayer on the sensing film was reported to exhibit sensitivity enhancement effects ${ }^{5}$. Attributed to the excellent optoelectronic properties and the advanced fabrication techniques (e.g. graphene growth on metallic substrates $)^{10,11}$, graphene layers have been employed to enhance the SPR biosensor sensitivity. As reported in Wu et al. ${ }^{12}$ study, 10 layers of the graphene coated on the gold sensing surface can improve the sensitivity by $25 \%$. The enhanced effect of single nanomaterial toppings, however, still seems insufficient for further development of SPR biosensor applications. Thus, hybrid nanostructures such as silicon-graphene and $\mathrm{MoS}_{2}$-graphene thin film were investigated and revealed prominent sensitivity enhanced effect ${ }^{13-15}$. The silicon nanosheet is able to enhance the sensitivity of the SPR biosensor because of its large real value of the refractive index. It also serves as a protective layer of metal film to improve the overall system stability ${ }^{16,17}$. Recently, the emerging two-dimensional (2D) transition metal dichalcogenides (TMDCs) have been widely used in transistors and photodetectors due to the remarkable electrical and optical properties. The TMDCs family consists of more than forty compounds that generally defined as $\mathrm{MX}_{2}$, where $\mathrm{M}$ stands for the transition metal from group IV to group VII, like $\mathrm{Nb}$, Ta, Mo and W; and the $\mathrm{X}$ denotes the chalcogen such as S, Se and Te. Monolayer $\mathrm{MX}_{2}$ contains three atomic layers where the transition metal layer is sandwiched by two chalcogens layers. Each layer is stacked via van der Waals forces. In this work, we focus on the group-IV semiconductor dichalcogenides $\mathrm{MoX}_{2}$ and $\mathrm{WX}_{2}$, namely Molybdenum disulfide $\left(\mathrm{MoS}_{2}\right)$, Molybdenum diselenide $\left(\mathrm{MoSe}_{2}\right)$, Tungsten disulfide $\left(\mathrm{WS}_{2}\right)$ and Tungsten diselenide $\left(\mathrm{WSe}_{2}\right)$. The rapid fabrication development of the high quality (i.e., large areas, highly uniform) individual $2 \mathrm{D} \mathrm{MX}_{2}$ layers by chemical exfoliation method promotes versatility of $2 \mathrm{D} \mathrm{MX}$ in various fields, such as photonics, electronics, energy storage, catalysis and even biomedical applications ${ }^{18-20}$. Although the properties of bulk $\mathrm{MX}_{2}$ have been investigated for decades, the successful translation of $2 \mathrm{D} \mathrm{MX}_{2}$ in optoelectronics and nanoelectronics is still remained in a stagnant stage. It is well known that when the bulk material is downscaled to a single layer, the bandgap transition would be gradually shifted from indirect to direct state ${ }^{20,21}$. This can be explained by the quantum confinement and resulted from the change in hybridization between orbital of Mo/W and X atoms ${ }^{20,22}$. The electronic bands of these monolayer materials are comparable to that of silicon $(1.1 \mathrm{eV})$, which allows good performance in digital transistors ${ }^{23}$. Furthermore, these characteristics also affect the photophysical properties. For the semiconductor materials with a direct bandgap like $\mathrm{MoX}_{2}$ and $\mathrm{WX}_{2}$, the photons can be directly absorbed or emitted if the external energy is larger than the bandgap. However, for the indirect bandgap materials, photons could not be absorbed directly, since additional phonons were needed to provide the energy for the electron to surpass the intermediate state and transfer the momentum to the crystal lattice. Therefore, the photon absorption process for direct bandgap materials are much more efficient than that of indirect bandgap materials.

The single layered $\mathrm{MoS}_{2}$, which known as "beyond graphene" 2D nanocrystals material has attracted a great deal of attention. Due to the quantum confinement effects, the monolayer $\mathrm{MoS}_{2}$ has a direct bandgap of $1.8 \mathrm{eV}$, while bulk $\mathrm{MoS}_{2}$ has an indirect bandgap of $1.2 \mathrm{eV}^{22,24}$. This allows $2 \mathrm{D} \mathrm{MoS}_{2}$ to be used in nano-transistor channel with a large switching ratio ${ }^{25}$ and in photodetectors with a high responsivity up to $5 \times 10^{8} \mathrm{AW}^{-126}$. Monolayer $\mathrm{MoS}_{2}$ also plays a key role for enhancing the sensitivity of SPR optical sensor ${ }^{13}$. Basically, a $\mathrm{MoS}_{2}$ enhanced hybrid nanostructure SPR biosensor can drastically improve detection limit of the device by using phase modulation technique. Similar to $\mathrm{MoS}_{2}$, the confinement of charge carriers on the horizontal atomic plane can gradually enlarge the energy gap of the $\mathrm{WS}_{2}$ atomic layers ${ }^{27-29}$. Theoretical studies show that monolayer $\mathrm{WS}_{2}$ has better performance compared to $\mathrm{MoS}_{2}$ in terms of enhancing the carrier mobility when they serve as channels in the transistor. This is attributed to the lower electron effective mass of $\mathrm{WS}_{2}$ compared to other $\mathrm{MX}_{2}$ materials $^{30}$. In the research field of photoelectronics, nano-scaled $\mathrm{WS}_{2}$ also showed outstanding performance. For example, $2 \mathrm{D}$ heterostructures consisting of $\mathrm{WS}_{2}, \mathrm{MoS}_{2}, \mathrm{GaSe}$ and graphene can exhibit photovoltaic effects with external quantum efficiencies up to $30 \%{ }^{27,31}$. Moreover, the employment of $\mathrm{WS}_{2}$ monolayer in plasmonic applications has enhanced the efficiency appreciably ${ }^{32}$. WSe $e_{2}$ nanolayers also attracted wide attention in photoelectronics, due to its fine absorption and emission features ${ }^{33}$ as well as the strong exciton charging effect ${ }^{34}$. Koperski et al. ${ }^{35}$ reported a comprehensive study of optical micro-spectroscopy based on thin layers of $\mathrm{WSe}_{2}$, where narrow emission lines $\left(\sim 100 \mu \mathrm{eV}\right.$ linewidth) were obtained because the monolayer $\mathrm{WSe}_{2}$ can generate luminescence within the same energy range. Ross et al. ${ }^{36}$ demonstrated a monolayer $\mathrm{WSe}_{2} \mathrm{p}-\mathrm{n}$ junction based LED structure that produced effective injections of electrons and holes due to the high optical quality. It yielded bright electroluminescence 


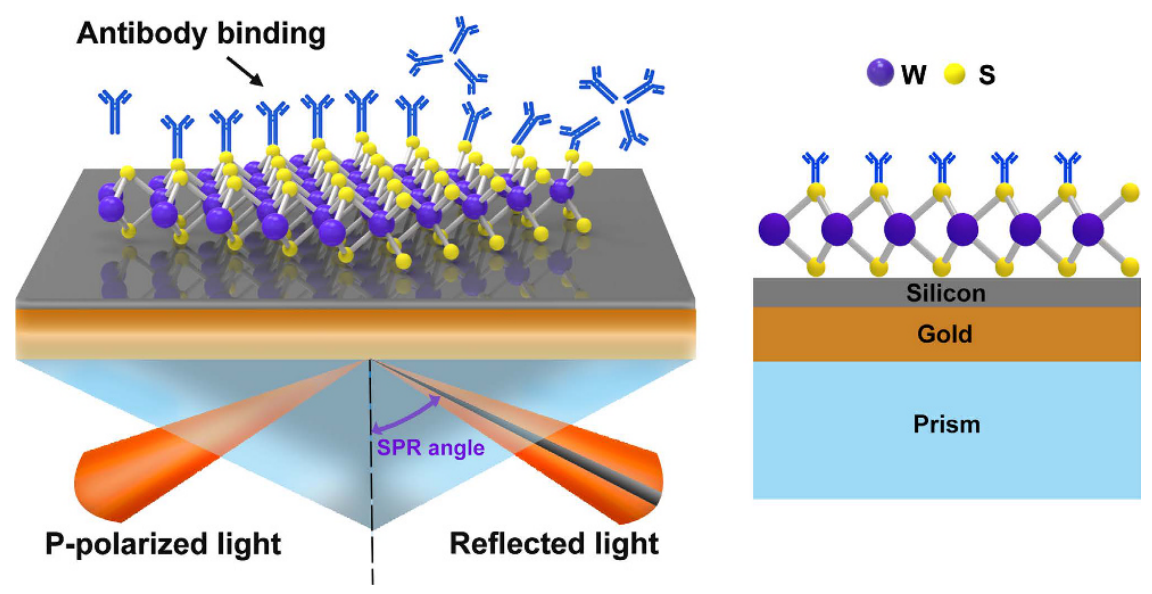

Figure 1. Schematic diagram of silicon- $\mathrm{WS}_{2} /$ nanosheets-enhanced surface plasmon resonance biosensor.

with much smaller injection current and linewidth compared with $\mathrm{MoS}_{2}$-based structures. The monolayer $\mathrm{MoSe}_{2}$ also provides promising optical applications because of its direct bandgap (identified at $1.55 \mathrm{eV})^{37}$. It is reported that the $\mathrm{MoSe}_{2}$ nanostructures show reversible and sensitive photo-responsive (PR) properties with the PR current values reaching up to $2.55 \times 10^{-5} \mathrm{~A}^{38}$. The lateral heterojunctions within monolayer $\mathrm{MoSe}_{2}-\mathrm{WSe}_{2}$ are visible under the optical microscope and show enhanced photoluminescence ${ }^{39}$. The strong photoluminescence emission is caused by the transition from an indirect band gap semiconductor of bulk material to a direct band gap semiconductor in atomically thin form ${ }^{40}$. These properties provided a solid foundation for atomic thin semiconductor dichalcogenides as promising candidates for next generation nanoelectronics, and optoelectronics ${ }^{41}$.

Based on these remarkable properties of silicon and group-IV semiconductor dichalcogenides, we propose a new configuration for sensitivity enhanced SPR biosensors based on silicon-MX $\mathrm{M}_{2}$ heterostructures. As shown in Fig. 1, based on the Kretschmann attenuated total reflection (ATR) configurations, gold thin film is attached at the bottom of the SF10 prism followed by silicon nanosheet and 2D MX. The 2D MX layers which are directly contacted with biomolecular analyte have dual effects: (i) as the signal-enhanced layer due to a high charge transfer efficiency from the $\mathrm{MX}_{2}$ surface to the Au surface ${ }^{42-44}$; (ii) as sensing platform to capture the biomolecules through the van der Waals interaction ${ }^{45,46}$.

\section{Results and Discussion}

In the ATR configuration, according to the principle of energy conversation the sum of the absorption $A$, reflectance $R$ and transmittance $T$ must be equal to 1 (i.e., $A+R+T=1$ ) assuming no energy loss besides the materials absorption. Under the ATR condition, $T$ is always equal to zero, hence the absorption of the system can be reduced to $A=1-R$. When the SPs were excited, the reflectance $R$ gradually decreased until it reached a minimum. The minimum $R$ was close to zero indicating that the incident energy was almost completely absorbed by the layered materials. Therefore, maximum incident light energy transfer to the evanescent wave is required in order to achieve the best SPR enhancement performance $e^{4,15,47,48}$.

Optimization of number of $\mathrm{MX}_{2}$ layers. To optimize the number of $\mathrm{MX}_{2}$ layers, we plotted the resonance depths (i.e., the value of minimum reflectivity) change as a function of number of $\mathrm{MX}_{2}$ layers with various thickness of silicon nanosheet (i.e., $0 \mathrm{~nm}, 5 \mathrm{~nm}, 7 \mathrm{~nm}$ ) and gold thin film (i.e., $30 \mathrm{~nm}, 35 \mathrm{~nm}, 40 \mathrm{~nm}, 50 \mathrm{~nm}$ ). Figures $2 \mathrm{a}-\mathrm{c}, 3 \mathrm{a}-\mathrm{c}, 4 \mathrm{a}-\mathrm{c}, 5 \mathrm{a}-\mathrm{c}$ and $6 \mathrm{a}-\mathrm{c}$ show the simulation results where different excitation wavelengths at $600 \mathrm{~nm}, 633 \mathrm{~nm}, 660 \mathrm{~nm}, 785 \mathrm{~nm}$ and $1024 \mathrm{~nm}$ were used. In general, two features for the SPR curves were observed: (i) When the silicon thickness was fixed and decreasing the gold thickness, the SPR dips redshifted with larger number of $\mathrm{MX}_{2}$ layers; similarly, when the thickness of gold thin film was fixed and decreasing the thickness of silicon, the reflectivity dips would also redshifted with the larger number of $\mathrm{MX}_{2}$ layers. This indicates that both silicon and $\mathrm{MX}_{2}$ layers can serve as absorption mediums and transfer energy to the gold thin film. (ii) It is worth noting that when the gold thickness was fixed at $50 \mathrm{~nm}$, no $\mathrm{MX}_{2}$ is required to achieve the minimum reflectivity dip since $50 \mathrm{~nm}$ is the optimized thickness of gold thin film based on the conventional Kretschmann ATR configuration ${ }^{6}$. By analyzing the relationship between reflectivity and the number of $\mathrm{MX}_{2}$ layers, optimization of the number of $\mathrm{MX}_{2}$ layers was possible by selecting the specific number of layers that corresponded to the minimum resonance depths (i.e., the value of reflectivity that closest to zero). The detailed optimized number of $\mathrm{MX}_{2}$ layers with minimum reflectivity $\min R$ less than 0.03 were listed in Tables S1-S20 (Supplementary Information).

Thickness Optimization of gold and silicon layers. The optimized thickness of gold thin film and silicon nanosheet were obtained from the optimization of the sensitivity and FWHM. The SPR sensitivity serves as the key characteristic of SPR biosensor is defined in equation. (13) (See the Methods section). When the refractive index change of the biomolecules analyte layer $\left(\Delta n_{b i o}=0.005\right)$ was fixed, the sensitivity was governed by the change in resonance angle that acquired before and after the adsorption of biomolecules on the surface of the $\mathrm{MX}_{2}$ layers surface. Upon investigation of the variation of change in the resonance angle as a function of the number of $\mathrm{MX}_{2}$ layers as shown in Figs $2 \mathrm{~d}-\mathrm{f}$ to $6 \mathrm{~d}-\mathrm{f}$ three main features were observed: (i) As the number of $\mathrm{MX}_{2}$ layers 

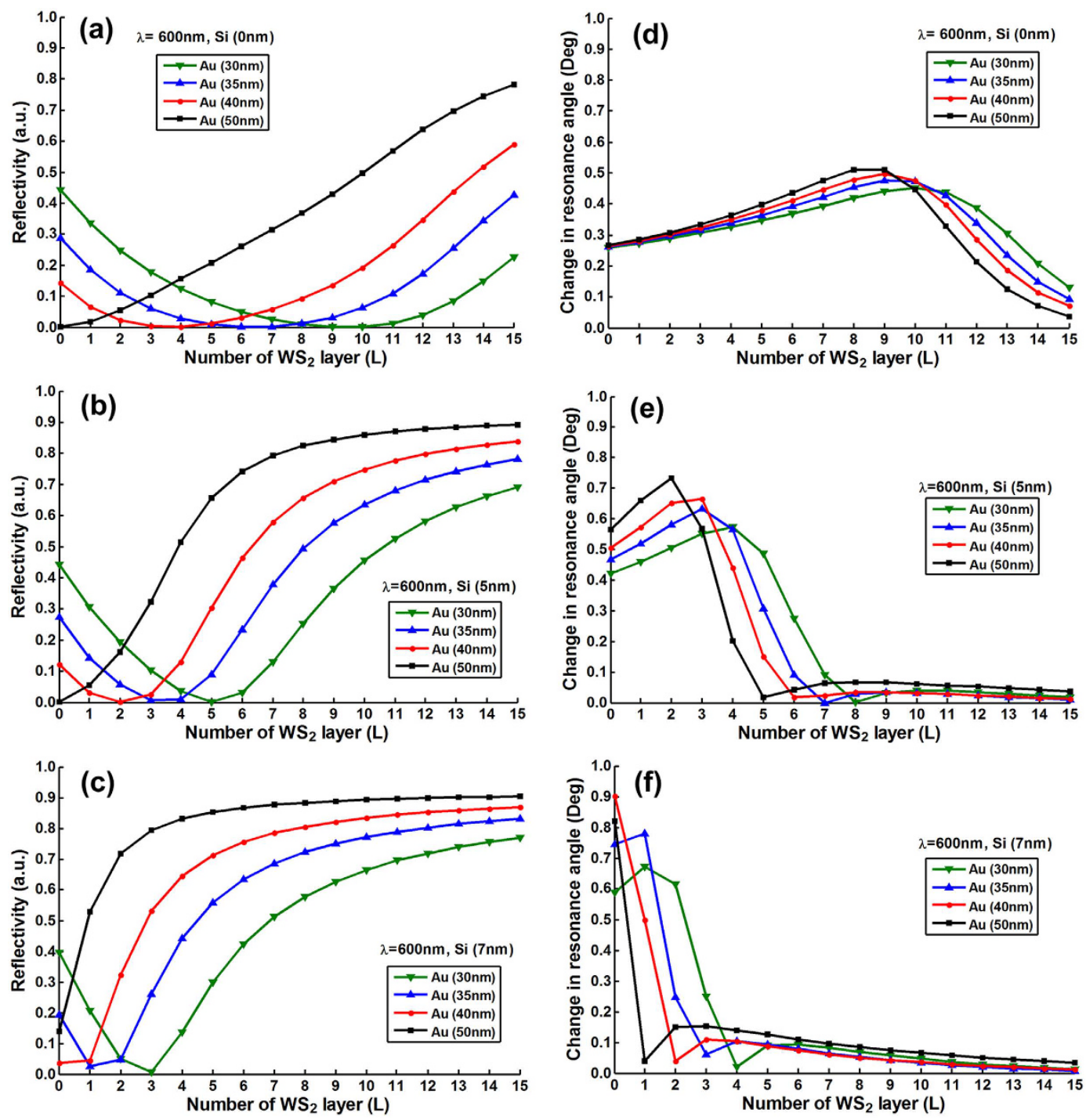

Figure 2. The minimum reflectivity in SPR curve as a function of the number of $\mathrm{WS}_{2}$ layers at $600 \mathrm{~nm}$ excitation wavelength with various thickness of the gold thin film and silicon nanosheet (a) $0 \mathrm{~nm}$ (b) $5 \mathrm{~nm}$ (c) $7 \mathrm{~nm}$; and the corresponding changes in the resonance angle for a fixed refractive index change in the biomolecular analyte $\left(\Delta n_{\text {bio }}=0.005\right)$ as a function of the number of layers of $\mathrm{WS}_{2}$ at $600 \mathrm{~nm}$ excitation wavelength with various thickness of gold thin film and silicon nanosheet (d) $0 \mathrm{~nm}$ (e) $5 \mathrm{~nm}(\mathbf{f}) 7 \mathrm{~nm}$.

increased, the change in resonance angle increased until it reached a maximum followed by a rapid decreasing to quasi-zero. (ii) When the excitation wavelength and gold thickness were fixed, the reduced thickness of silicon nanosheet caused the peak of the change in resonance angle to shift toward a larger number of $\mathrm{MX}_{2}$ layers; similarly, if the excitation wavelength and the silicon thickness were fixed, the decreased thickness of gold thin film also led to a peak shift to a larger number of $\mathrm{MX}_{2}$ layers. (iii) With fixed thickness of silicon and gold, the redshifted excitation wavelengths induced the peak shift of change in resonance angle towards the larger number of $\mathrm{MX}_{2}$ layers. Similar behaviors were also observed in Figs $2 \mathrm{a}-\mathrm{c}$ to $6 \mathrm{a}-\mathrm{c}$ (i.e., at longer excitation wavelengths, a larger number of $\mathrm{MX}_{2}$ was required to achieve the minimum reflectivity dip). This showed that the angular sensitivity decreases with longer excited wavelength ${ }^{5}$, thereby higher refractive index materials are required to enhance the evanescent field.

With optimized number of $\mathrm{MX}_{2}$ layers, the angular sensitivity and FWHM of the four silicon-MX $\mathrm{M}_{2}$ enhanced models were then analyzed under five different excitation wavelengths. When the excitation wavelength was set at $600 \mathrm{~nm}$, the highest sensitivity $155.68 \mathrm{Deg} / \mathrm{RIU}$ was achieved with optimum parameters of $35 \mathrm{~nm}$ thickness gold film, $7 \mathrm{~nm}$ thickness silicon and monolayer $\mathrm{WS}_{2}$. The FWHM was 17.4644 Deg with a minimum reflectivity of $2.5592 \times 10^{-2}$ as shown in Table S1 (Supplementary Information). However, with the same thickness parameters and excitation wavelength, the highest sensitivity of silicon-MoSe ${ }_{2}$ enhanced model was only 104.56 Deg/RIU with a minimum reflectivity of $4.7627 \times 10^{-3}$ as shown in Table S6 (Supplementary Information). This result can be explained by the different optical properties of $\mathrm{WS}_{2}$ and $\mathrm{MoSe}_{2}$. At $600 \mathrm{~nm}$ excitation wavelength, the real part of the dielectric constant of $\mathrm{MoSe}_{2}$ is 2 times as that of $\mathrm{WS}_{2}$, which indicates $\mathrm{MoSe}_{2}$ layers have higher energy 

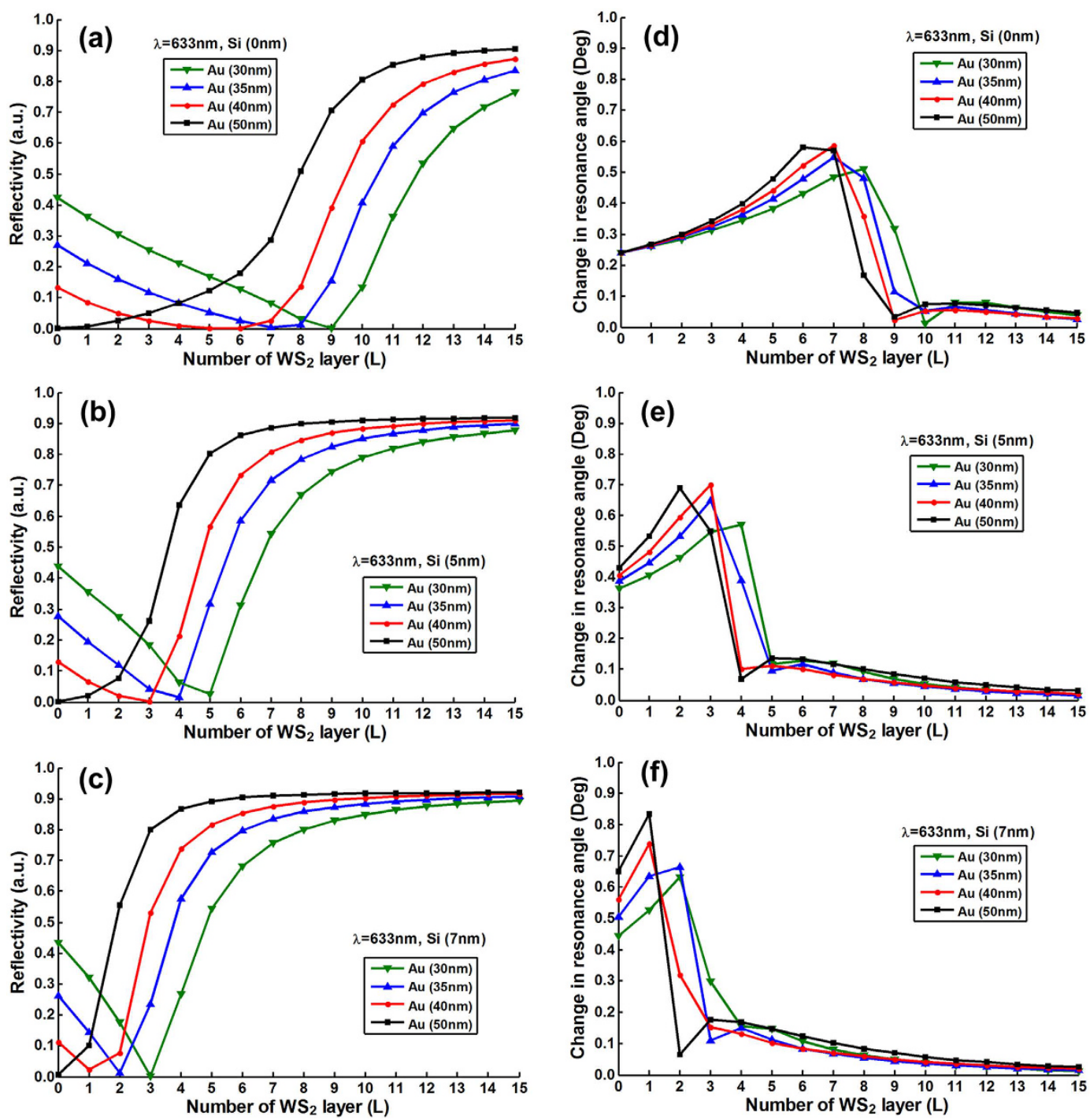

Figure 3. The minimum reflectivity in SPR curve as a function of the number of $\mathrm{WS}_{2}$ layers at $633 \mathrm{~nm}$ excitation wavelength with various thickness of the gold thin film and silicon nanosheet (a) $0 \mathrm{~nm}$ (b) $5 \mathrm{~nm}$ (c) $7 \mathrm{~nm}$; and the corresponding changes in the resonance angle for a fixed refractive index change in the biomolecular analyte $\left(\Delta n_{b i o}=0.005\right)$ as a function of the number of layers of $\mathrm{WS}_{2}$ at $633 \mathrm{~nm}$ excitation wavelength with various thickness of gold thin film and silicon nanosheet (d) $0 \mathrm{~nm}(\mathbf{e}) 5 \mathrm{~nm}(\mathbf{f}) 7 \mathrm{~nm}$.

absorption compared to $\mathrm{WS}_{2}$ layers. As a result, the $\operatorname{minR}$ of silicon-MoSe $\mathrm{e}_{2}$ enhanced model is lower than that of silicon-WS $\mathrm{W}_{2}$. However, the absorbed energy is not completely transferred to enhance the evanescent field due to the energy loss during the process. It is known that a dielectric material with a large real part and a small image part of the dielectric function have low energy loss, therefore the $\mathrm{WS}_{2}$ layers have much lower energy loss than that of $\mathrm{MoSe}_{2}$. Furthermore, the penetration depth of the evanescent field in the biomolecular analyte layer of the silicon- $\mathrm{WS}_{2}$ model is deeper than that of the silicon-MoSe $\mathrm{H}_{2}$ model, since the real part of dielectric constant of $\mathrm{WS}_{2}$ is smaller than $\mathrm{MoSe}_{2}$ [6]. Consequently, the evanescent field of silicon-WS $\mathrm{W}_{2}$ model is more sensitive than that of silicon- $\mathrm{MoSe}_{2}$ model to the refractive index change in biomolecular analyte. All these factors contributed to higher sensitivity in the silicon-WS $\mathrm{W}_{2}$ enhanced scheme at $600 \mathrm{~nm}$ excitation wavelength. Similarly, the thickness combination for excitation wavelengths ranging from $633 \mathrm{~nm}$ to $1024 \mathrm{~nm}$ were also optimized (see Supplementary Information Tables S2-S20). Based on these results, we could conclude that although the $\mathrm{MX}_{2}$ layers with large real values of the dielectric constant contributed to the increased energy absorption, the intrinsic energy loss in these layers played a more significant role in the sensitivity of the multi-layered system.

Influence of excitation wavelength. In order to obtain the best SPR sensing performance, it is also important to achieve a relatively low FWHM as it promises more accurate determination of the angular modulation. The value of the FWHM is mainly depends on two factors: excitation wavelength and the number of $\mathrm{MX}_{2}$ layers. As reported previously, using longer excitation wavelength results in a narrower resonance curve ${ }^{6}$. As for the latter factor, our simulation results showed that large number of $\mathrm{MX}_{2}$ layers generated a higher value 

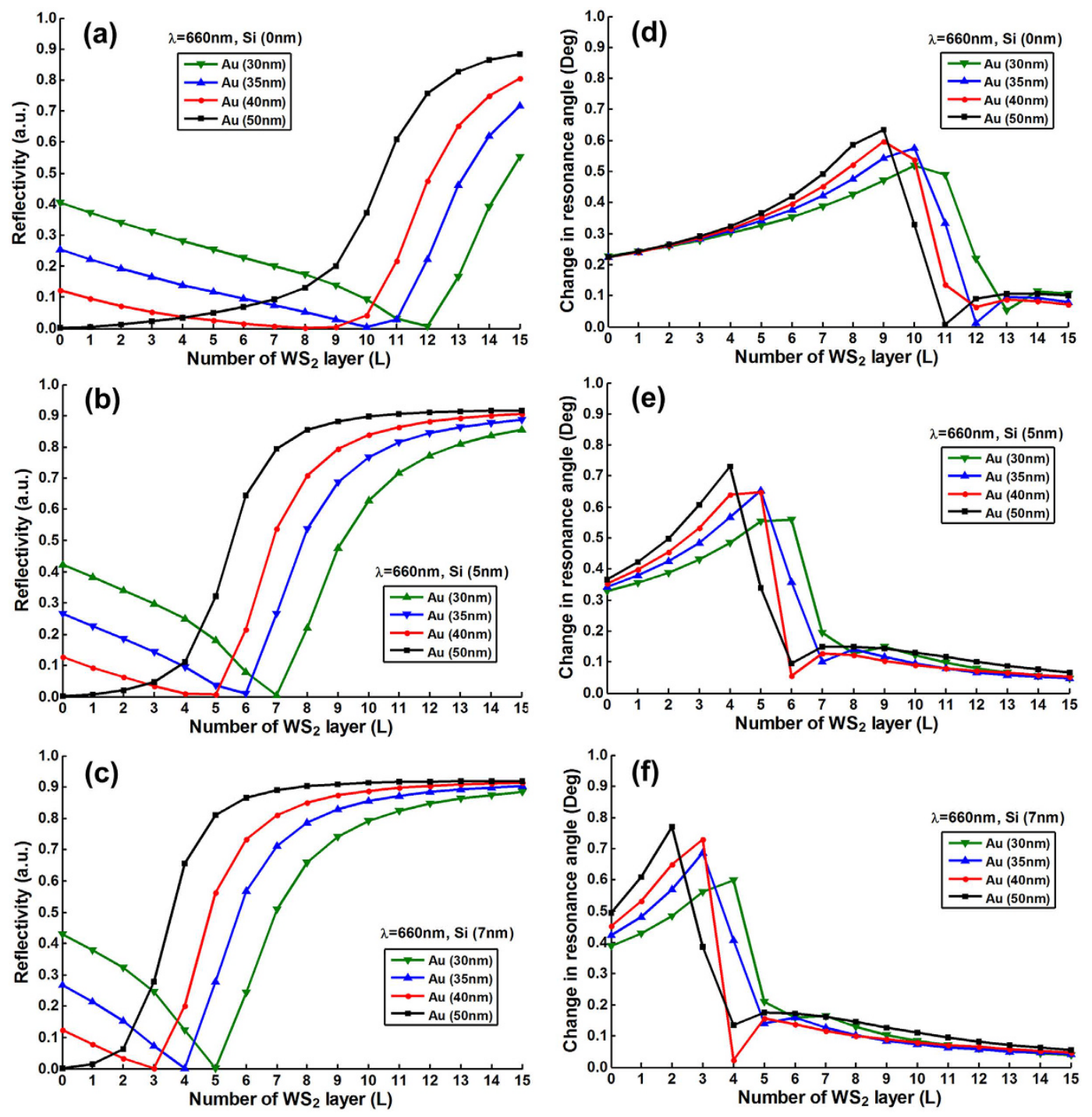

Figure 4. The minimum reflectivity in SPR curve as a function of the number of $\mathrm{WS}_{2}$ layers at $660 \mathrm{~nm}$ excitation wavelength with various thickness of the gold thin film and silicon nanosheet (a) $0 \mathrm{~nm}$ (b) $5 \mathrm{~nm}$ (c) $7 \mathrm{~nm}$; and the corresponding changes in the resonance angle for a fixed refractive index change in the biomolecular analyte $\left(\Delta n_{\text {bio }}=0.005\right)$ as a function of the number of layers of $\mathrm{WS}_{2}$ at $660 \mathrm{~nm}$ excitation wavelength with various thickness of gold thin film and silicon nanosheet (d) $0 \mathrm{~nm}$ (e) $5 \mathrm{~nm}(\mathbf{f}) 7 \mathrm{~nm}$.

of FWHM since the additional $\mathrm{MX}_{2}$ layers resulted in higher electron energy loss and reduced the accuracy. To investigate the effect of the excitation wavelength, we summarized the optimized thickness combinations of gold thin film and silicon nanosheet for five different excitation wavelengths. As shown in Table 1, three features were observed: (i) By using $600 \mathrm{~nm}, 633 \mathrm{~nm}, 660 \mathrm{~nm}$ and $785 \mathrm{~nm}$ excitation wavelengths, the best performances were all achieved in the silicon-WS $\mathrm{W}_{2}$ sensing model except for the $1024 \mathrm{~nm}$ excitation wavelength which was attained in the silicon- $\mathrm{MoS}_{2}$ scheme. This was due to the hybrid effects of the energy absorption and energy loss of $\mathrm{MX}_{2}$ layers at the different excitation wavelengths. (ii) For the visible range, the optimized thickness of silicon was $7 \mathrm{~nm}$ and the $\mathrm{WS}_{2}$ layers were required to be ultra-thin (i.e., 1-3 layers), the predicted sensitivity were all above $140 \mathrm{Deg} / \mathrm{RIU}$. However, for the near-infrared range (i.e., $785 \mathrm{~nm}$ and $1024 \mathrm{~nm}$ ), the optimized thickness of silicon and gold were determined to be $5 \mathrm{~nm}$ and $40 \mathrm{~nm}$ respectively, with the optimized number of $\mathrm{MX}_{2}$ layers being at least 13 and above. (iii) It is worth noting that as the excitation wavelength redshifted, the optimized sensitivity decreased gradually. The dielectric constant changes significantly with the increasing incident wavelengths. The real part of the dielectric constant is related to the reflectivity of the interface while the imaginary part is indicative of the energy absorption. Therefore, according to Table 1, as the wavelengths progressed into the near-infrared region, the hybrid effects resulted in higher attenuation of the evanescent field and thus lower SPR sensitivity was observed ${ }^{49}$.

Optimized scheme for each of the Silicon- $\mathrm{MX}_{2}$ model. Finally, the parameters yielding the best performance for each of the silicon-WS 2 , silicon-WSe 2 , silicon- $\mathrm{MoS}_{2}$, silicon-MoSe $\mathrm{H}_{2}$ enhanced SPR models are 

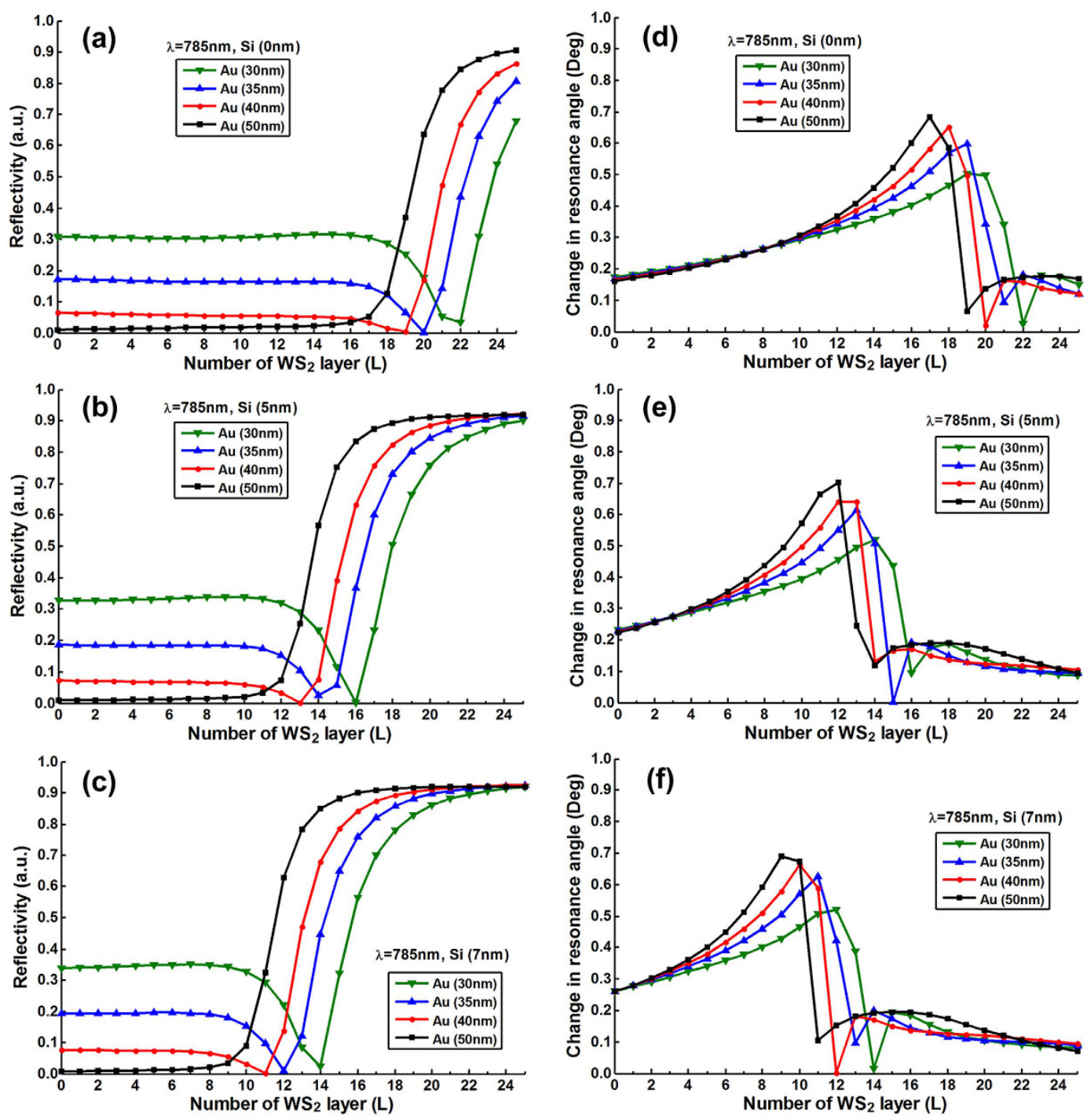

Figure 5. The minimum reflectivity in SPR curve as a function of the number of $\mathrm{WS}_{2}$ layers at $785 \mathrm{~nm}$ excitation wavelength with various thickness of the gold thin film and silicon nanosheet (a) $0 \mathrm{~nm}$ (b) $5 \mathrm{~nm}$ (c) $7 \mathrm{~nm}$; and the corresponding changes in the resonance angle for a fixed refractive index change in the biomolecular analyte $\left(\Delta n_{\text {bio }}=0.005\right)$ as a function of the number of layers of $\mathrm{WS}_{2}$ at $785 \mathrm{~nm}$ excitation wavelength with various thickness of gold thin film and silicon nanosheet (d) $0 \mathrm{~nm}(\mathbf{e}) 5 \mathrm{~nm}(\mathbf{f}) 7 \mathrm{~nm}$.

presented in Table 2. The optimized excitation wavelength and thickness of silicon nanosheet, gold thin film are the same for all the silicon- $\mathrm{MX}_{2}$ enhanced models except for the silicon-WS $\mathrm{W}_{2}$ model. For the silicon- $\mathrm{WS}_{2}$ scheme, it possessed the highest sensitivity of $155.68 \mathrm{Deg} / \mathrm{RIU}$ with $35 \mathrm{~nm}$ thick gold and $7 \mathrm{~nm}$ silicon at the $600 \mathrm{~nm}$ wavelength. The 3D plots shown in Fig. 7a-d further validates the optimized parameters by depicting the sensitivity as a function of number of $\mathrm{MX}_{2}$ layers and gold thickness with the corresponding optimized silicon thickness and excitation wavelengths. From Fig. $7 \mathrm{~b}-\mathrm{d}$, the highest value of sensitivity seemed to be attainable with $50 \mathrm{~nm}$ thick gold thin film and monolayer $\mathrm{MX}_{2}$. However, the minimum SPR reflectivity minR under these condition were greater than 0.03 , leading to low energy-transfer efficiencies. In addition, the slopes of the sensitivity as a function of $\mathrm{MX}_{2}$ layers coated on $40 \mathrm{~nm}$-thickness $\mathrm{Au}$ thin film were the sharpest among all the other Au thicknesses. Therefore, we concluded that the highest sensitivity of silicon- $\mathrm{MoS}_{2}$, silicon- $\mathrm{MoSe}_{2}$ and silicon-WSe $\mathrm{C}_{2}$ models were obtained with $40 \mathrm{~nm}$ thick gold films. We also compared the sensitivity of our optimized configuration with the well-known conventional Kretschmann configuration (with $50 \mathrm{~nm}$ thickness gold thin film). As shown in Fig. 8, the sensitivity of our optimized silicon-WS $\mathrm{WS}_{2}$ enhanced configuration (155.68 Deg/RIU as shown in red solid line) is 3 times more sensitive than that of the Kretschmann design (53.40 Deg/RIU as shown in blue dashed line). For the Kretschmann configuration without any dielectric layers, the only absorbing medium in the system is the metal film; whereas in the silicon- $\mathrm{WS}_{2}$ enhanced structure the additional silicon nanosheet and $\mathrm{MX}_{2}$ layers can serve as the absorption medium as well. Moreover, the high refractive index of silicon nanosheet and the high real part of $\mathrm{MX}_{2}$ dielectric constant can enhance the evanescent field at the metal interface ${ }^{49}$. These double effects result in drastic sensitivity enhancement in the silicon-WS $\mathrm{W}_{2}$ enhanced structure. In order to 

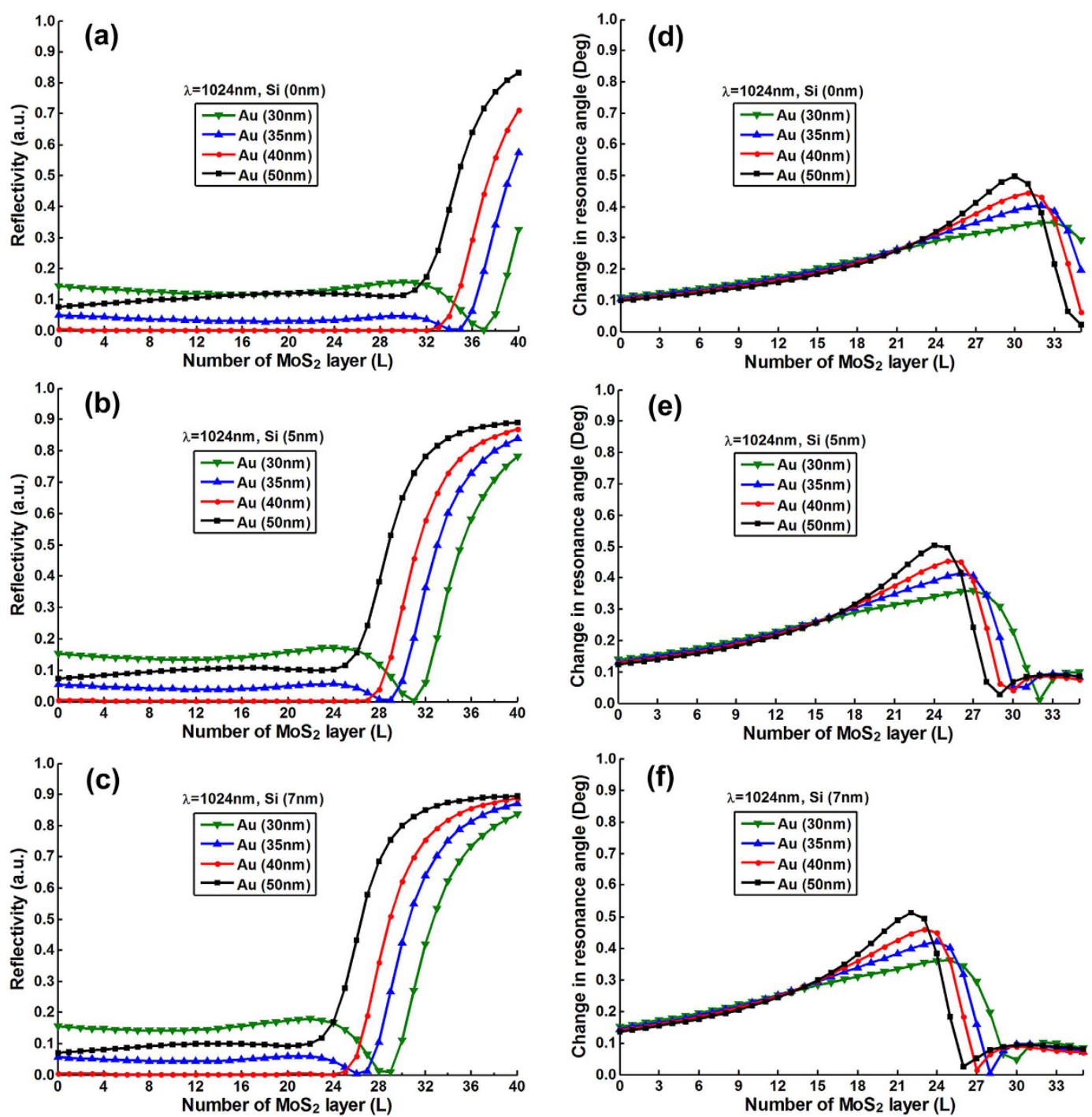

Figure 6. The minimum reflectivity in SPR curve as a function of the number of $\mathrm{WS}_{2}$ layers at $1024 \mathrm{~nm}$ excitation wavelength with various thickness of the gold thin film and silicon nanosheet (a) $0 \mathrm{~nm}$ (b) $5 \mathrm{~nm}$ (c) $7 \mathrm{~nm}$; and the corresponding changes in the resonance angle for a fixed refractive index change in the biomolecular analyte $\left(\Delta n_{b i o}=0.005\right)$ as a function of the number of layers of $\mathrm{WS}_{2}$ at $1024 \mathrm{~nm}$ excitation wavelength with various thickness of gold thin film and silicon nanosheet (d) $0 \mathrm{~nm}$ (e) $5 \mathrm{~nm}(\mathbf{f}) 7 \mathrm{~nm}$.

\begin{tabular}{|c|c|c|c|c|c|c|c|c|}
\hline $\begin{array}{l}\text { Excitation } \\
\text { wavelength } \\
(\mathbf{n m})\end{array}$ & $\begin{array}{l}\text { Type of } \\
\text { TMDC }\end{array}$ & $\begin{array}{c}\text { Gold } \\
\text { thickness } \\
(\mathrm{nm})\end{array}$ & $\begin{array}{c}\text { Silicon } \\
\text { thickness } \\
(\mathrm{nm})\end{array}$ & $\begin{array}{c}\text { Number of } \\
\mathrm{WS}_{2} \text { layers } \\
(L)\end{array}$ & $\begin{array}{l}\text { Minimum } \\
\text { Reflectivity }\end{array}$ & $\begin{array}{c}\Delta \theta_{S P R}(\mathrm{Deg}) \\
\left(\Delta n_{b i o}=0.005\right)\end{array}$ & $\begin{array}{l}\text { Sensitivity } \\
\text { (Deg/RIU) }\end{array}$ & $\begin{array}{c}\text { FWHM } \\
\text { (Deg) }\end{array}$ \\
\hline 600 & $\mathrm{WS}_{2}$ & 35 & 7 & 1 & $2.5592 \times 10^{-2}$ & 0.7784 & 155.68 & 17.4644 \\
\hline 633 & $\mathrm{WS}_{2}$ & 40 & 7 & 1 & $2.4099 \times 10^{-2}$ & 0.7394 & 147.88 & 16.2417 \\
\hline 660 & $\mathrm{WS}_{2}$ & 40 & 7 & 3 & $3.3778 \times 10^{-5}$ & 0.7282 & 145.64 & 16.8245 \\
\hline 785 & $\mathrm{WS}_{2}$ & 40 & 5 & 13 & $2.5767 \times 10^{-3}$ & 0.6395 & 127.90 & 15.5232 \\
\hline 1024 & $\mathrm{MoS}_{2}$ & 40 & 5 & 26 & $1.6573 \times 10^{-6}$ & 0.4499 & 89.98 & 17.5445 \\
\hline
\end{tabular}

Table 1. The optimized values of gold thin film, silicon nanosheet thickness and the number of $\mathrm{MX}_{2}$ layers with corresponding changes in resonance angle, sensitivities and FWHMs in SPR curves for $600 \mathrm{~nm}$, $633 \mathrm{~nm}, 660 \mathrm{~nm}, 785 \mathrm{~nm}$ and $1024 \mathrm{~nm}$ excitation wavelengths.

demonstrate the validity of our N-layer 2D models, we have experimentally tested the SPR sensing ability with 3-layer graphene-coated Au thin film. And both the angular and phase measurement results matched well with our theoretical analyses (see Supplementary Figs S1-S3). 


\begin{tabular}{|c|c|c|c|c|c|c|c|c|}
\hline $\begin{array}{l}\text { Excitation } \\
\text { wavelength } \\
(\mathrm{nm})\end{array}$ & $\begin{array}{l}\text { Type of } \\
\text { TMDC }\end{array}$ & $\begin{array}{c}\text { Gold } \\
\text { thickness } \\
(\mathrm{nm})\end{array}$ & $\begin{array}{l}\text { Silicon } \\
\text { thickness } \\
(\mathbf{n m})\end{array}$ & $\begin{array}{l}\text { Number of } \\
W_{2} \text { layers } \\
(L)\end{array}$ & $\begin{array}{l}\text { Minimum } \\
\text { Reflectivity }\end{array}$ & $\begin{array}{c}\Delta \theta_{S P R}(\mathrm{Deg}) \\
\left(\Delta n_{b i o}=0.005\right)\end{array}$ & $\begin{array}{l}\text { Sensitivity } \\
\text { (Deg/RIU) }\end{array}$ & $\begin{array}{c}\text { FWHM } \\
\text { (Deg) }\end{array}$ \\
\hline 600 & $\mathrm{WS}_{2}$ & 35 & 7 & 1 & $2.5592 \times 10^{-2}$ & 0.7784 & 155.68 & 17.4644 \\
\hline 633 & $\mathrm{MoS}_{2}$ & 40 & 7 & 1 & $1.1513 \times 10^{-5}$ & 0.6586 & 131.70 & 17.5728 \\
\hline 633 & $\mathrm{WSe}_{2}$ & 40 & 7 & 2 & $5.3807 \times 10^{-3}$ & 0.7070 & 141.40 & 17.2340 \\
\hline 633 & $\mathrm{MoSe}_{2}$ & 40 & 7 & 1 & $2.0438 \times 10^{-3}$ & 0.6584 & 131.68 & 17.0915 \\
\hline
\end{tabular}

Table 2. The optimized values of gold thin film, silicon nanosheet thickness and the number of $\mathrm{MX}_{2}$ layers with corresponding excitation wavelength, change in resonance angle, sensitivity and FWHM of the SPR curve for each silicon- $\mathrm{MX}_{2}$ nanosheet enhanced model.

(a)

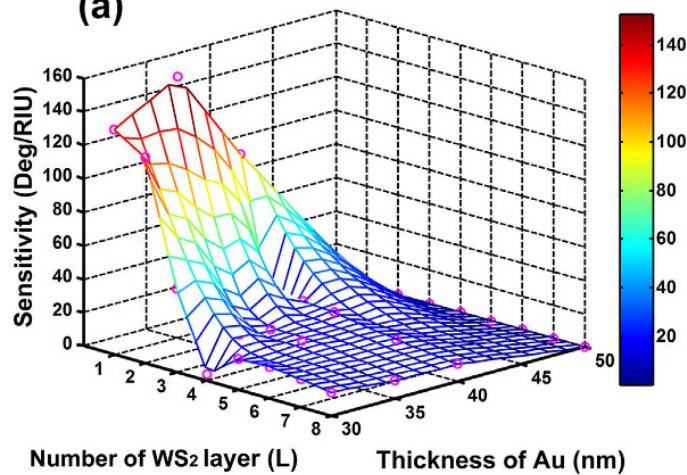

(c)

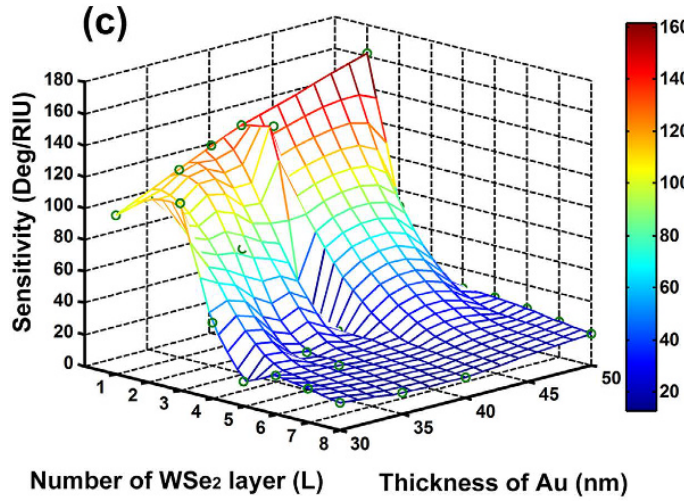

(b)

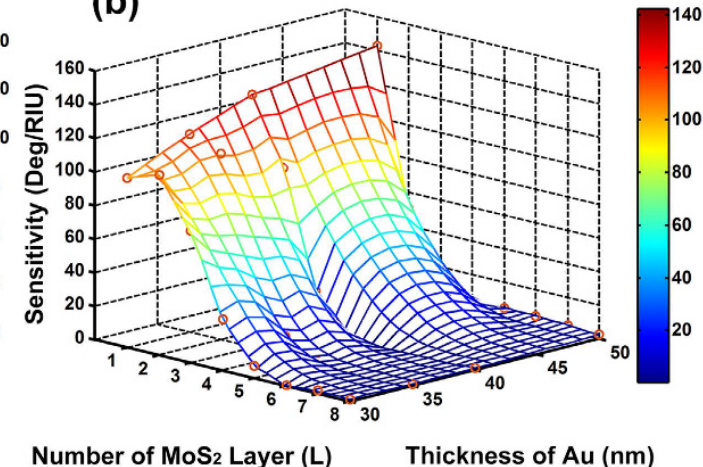

(d)

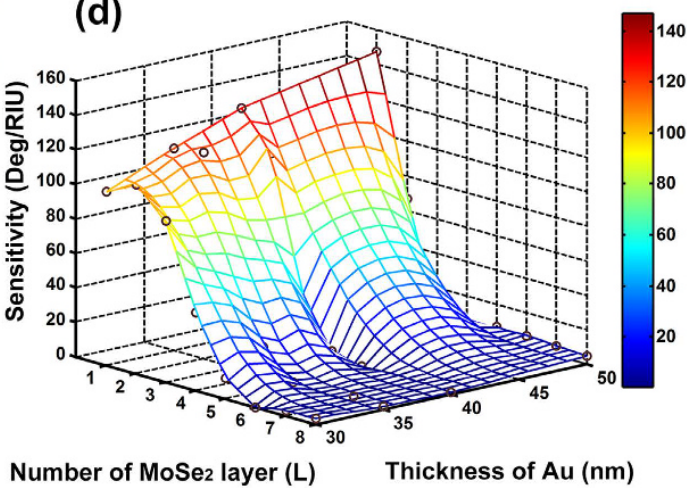

Figure 7. The variation of the sensitivity as a function of gold thin film thickness and number of $\mathrm{MX}_{2}$ layers with $7 \mathrm{~nm}$ thick silicon nanosheet. (a) The excitation wavelength is $600 \mathrm{~nm}$ in silicon-WS $\mathrm{W}_{2}$ nanosheet enhanced model; (b) The excitation wavelength is $633 \mathrm{~nm}$ in silicon- $\mathrm{MoS}_{2}$ nanosheet enhanced model; (c) The excitation wavelength is $633 \mathrm{~nm}$ in silicon-WSe ${ }_{2}$ nanosheet enhanced model; (d) The excitation wavelength is $633 \mathrm{~nm}$ in silicon- $\mathrm{MoSe}_{2}$ nanosheet enhanced model.

\section{Conclusion}

In this study, we demonstrated a silicon nanosheet and 2D MX $\mathrm{MX}_{2}$ enhanced surface plasmon resonance biosensor. Based on the Kretschmann configuration, the system consists of SF10 triangular prism, gold thin film, silicon nanosheet and $2 \mathrm{D} \mathrm{MX}$ film $\left(\mathrm{MoS}_{2} / \mathrm{MoSe}_{2} / \mathrm{WS}_{2} / \mathrm{WSe}_{2}\right)$. To investigate the enhancement effect of each $\mathrm{MX}_{2}$ materials, we designed four enhanced models, namely, silicon-WS , silicon-WSe $e_{2}$, silicon- $\mathrm{MoS}_{2}$ and silicon-MoSe 2 . Maxwell's equations, Fresnel equations and transfer matrix method were used to analyze the change in resonance angle and the corresponding sensitivity for angular modulation. To study the influence of the excitation wavelengths to sensing performance, we studied five different excitation wavelengths, namely, $600 \mathrm{~nm}, 633 \mathrm{~nm}$, $660 \mathrm{~nm}, 785 \mathrm{~nm}$ and $1024 \mathrm{~nm}$. The results showed that the silicon nanosheet together with each of the four types of $2 \mathrm{D} \mathrm{MX}_{2}$ layers could compensate the SPR effect of gold and significantly improve the sensitivity of the biosensor. However, excessive $\mathrm{MX}_{2}$ layers would result in increased energy loss and reduced the sensitivity. Therefore, in order to optimize the sensitivity, the thickness of gold film, silicon nanosheet and $\mathrm{MX}_{2}$ layers were investigated to minimize the reflectance and width of SPR curve to achieve a system with higher angular scanning accuracy. The combination of the optimized parameters for each excitation wavelength and each silicon-MX $\mathrm{MX}_{2}$ enhanced model were also presented with the highest SPR sensitivity of $155.68 \mathrm{Deg} / \mathrm{RIU}$ achieved using $35 \mathrm{~nm}$ thick gold film, $7 \mathrm{~nm}$ thick silicon nanosheet and a monolayer $\mathrm{WS}_{2}$ under the illumination of a $600 \mathrm{~nm}$ excitation wavelength source. 


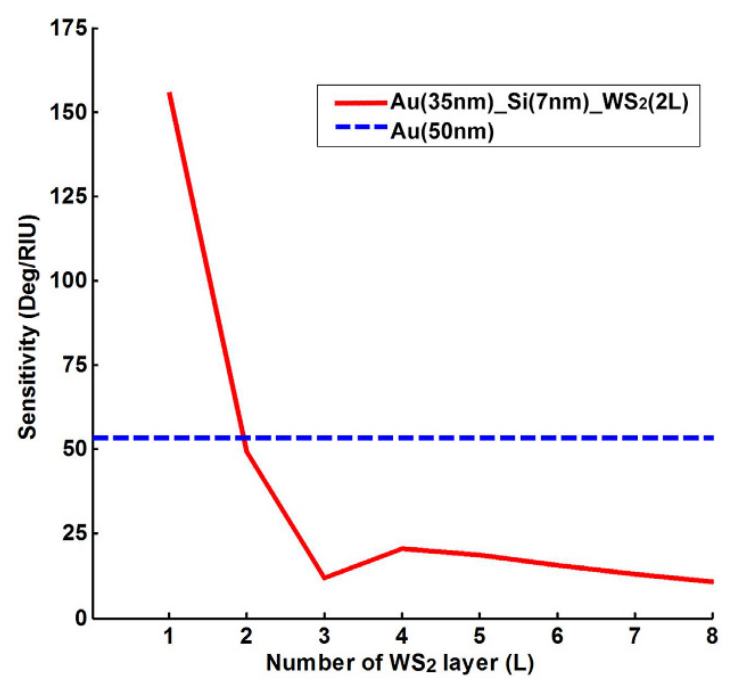

Figure 8. Comparison of the SPR sensing performances between the optimized scheme of $35 \mathrm{~nm}$ thick gold thin film, $7 \mathrm{~nm}$ thick silicon nanosheet and monolayer $\mathrm{WS}_{2}$ (the red solid line) and the conventional Kretschmann configuration with $50 \mathrm{~nm}$ thick Au substrate (the blue dashed line).

\section{Methods}

The wavelength-dependent refractive index for each layer. In this study, the theoretical analyses were performed with excitation light wavelengths in visible and near infrared ranges. Five wavelengths were chosen as $600 \mathrm{~nm}, 633 \mathrm{~nm}, 660 \mathrm{~nm}, 785 \mathrm{~nm}$ and $1024 \mathrm{~nm}$ respectively. The proposed configuration consists of 6 layer components, namley the SF10 prism, gold thin film, silicon nanosheet, $2 \mathrm{D} \mathrm{MX}$ nanolayers $\left(\mathrm{MoS}_{2} / \mathrm{WS}_{2} / \mathrm{MoSe}_{2} /\right.$ $\mathrm{WSe}_{2}$ ), biomolecular analyte layer and sensing medium layer. The first component is the SF10 prism whose refractive index is given $b^{50}$ :

$$
n_{\text {prism }}(\lambda)=\left(\frac{1.62153902 \lambda^{2}}{\lambda^{2}-0.0122241457}+\frac{0.256287842 \lambda^{2}}{\lambda^{2}-0.0595736775}+\frac{1.64447552 \lambda^{2}}{\lambda^{2}-147.468793}+1\right)^{1 / 2}
$$

where $\lambda$ represents the wavelength of the light source in $\mu \mathrm{m}$ and Eq. (2) is valid for wavelengths from $0.38 \mu \mathrm{m}$ to $2.5 \mu \mathrm{m}$. The refractive index of the gold thin film is determined using the Drude model by:

$$
n_{\text {gold }}(\lambda)=\left(\varepsilon^{\prime}{ }_{\text {gold }}+i \varepsilon^{\prime \prime}{ }_{\text {gold }}\right)^{1 / 2}=\left(1-\frac{\lambda^{2} \lambda_{c}}{\lambda_{p}^{2}\left(\lambda_{c}+\mathrm{i} \lambda\right)}\right)^{1 / 2}
$$

where $\lambda_{p}\left(1.6826 \times 10^{-7} \mathrm{~m}\right)$ denotes the plasma wavelength and $\lambda_{c}\left(8.9342 \times 10^{-6} \mathrm{~m}\right)$ is the collision wavelength. $\lambda$ in Eq. (3) represents the wavelength of the light source in $\mu \mathrm{m}$, which is valid from $0.18 \mu \mathrm{m}$ to $1.94 \mu \mathrm{m}^{51-53}$. The thickness of the gold thin film was varied from $30 \mathrm{~nm}$ to $50 \mathrm{~nm}$ in this study. The refractive index of the silicon nanosheet is calculated by:

$$
n_{\text {silicon }}(\lambda)=A+A_{1} e^{-\lambda / t_{1}}+A_{2} e^{-\lambda / t_{2}}
$$

where $A=3.44904, A_{1}=2271.88813, A_{2}=3.39538, t_{1}=0.058304$ and $t_{2}=0.30384$, and $\lambda$ is the wavelength in $\mu \mathrm{m}^{54}$. The thickness of the silicon sheet was varied from $0 \mathrm{~nm}$ to $7 \mathrm{~nm}$. The fourth layer is the core functional layer $-2 \mathrm{D}$ $\mathrm{MX}_{2}\left(\mathrm{MoS}_{2} / \mathrm{WS}_{2} / \mathrm{MoSe}_{2} / \mathrm{WSe}_{2}\right)$ whose refractive indexs and the monolayer thickness $t_{M X 2}$ are summarised in Table $3^{55,56}$. The thickness of MX 2 layers $d_{M X 2}$ is described by $d_{M X 2}=t_{M X 2} \times L$, where $L$ represents the number of $\mathrm{MX}_{2}$ layers. The fifth layer is the biomolecular analyte that dissolved in sensing medium. The change in refractive index of this layer can be induced by absorption of the biomolecules on the $\mathrm{MX}_{2}$ surface. Here the refractive index of this layer can be represented as $n_{\text {analyte }}=1.330+\Delta n_{b i o}$, where $\Delta n_{b i o}$ indicates the change in refractive index of the biomolecular analyte. The thickness of this layer $d_{\text {analyte }}$ is fixed at $100 \mathrm{~nm}$. The last layer is the sensing medium, the refractive index is defined as $n_{\text {solvent }}=1.330$.

Reflection coefficient $\left(r_{p}\right)$ and Reflectivity $\left(R_{p}\right)$. In this study, the SPR biosensor model is based on the well-known Kretchmann's attenuated total reflection (ATR) structure. The incident light passes through the prism and is totally reflected at the base of the prism. Most of the light energy is absorbed by the metal and dielectric layers to generate the evanescent wave which propagates along the interface and penetrates into the sensing film. When the evanescent wave vector $k_{x}$ matches with the surface plasmon wave vector $k_{s p}$, the resonance occurs as shown in Eq. (1). In order to analyse our N-layer structure model, the transfer matrix method (TMM) was employed. Each layer in our system was stacked horizontally in z-axis direction. All layers are defined by the parameters $n_{k}, \varepsilon_{k}$ and $d_{k}$, which represent the refractive index, dielectric constant and thickness of the $k_{\mathrm{th}}$ layer, respectively. $\theta_{k}$ denotes the incident angle of the $k_{\mathrm{th}}$ layer and $\lambda$ stands for the excitation wavelength. 


\begin{tabular}{|l|c|c|c|c|c|c|}
\hline TMDC & $\boldsymbol{T}_{M X 2}(\mathbf{n m})$ & $\boldsymbol{\lambda}=\mathbf{6 0 0} \mathbf{n m}$ & $\boldsymbol{\lambda}=\mathbf{6 3 3} \mathbf{n m}$ & $\boldsymbol{\lambda}=\mathbf{6 6 0} \mathbf{n m}$ & $\boldsymbol{\lambda}=\mathbf{7 8 5} \mathbf{n m}$ & $\boldsymbol{\lambda}=\mathbf{1 0 2 4} \mathbf{n m}$ \\
\hline \multirow{2}{*}{$\mathrm{MoS}_{2}$} & \multirow{2}{*}{0.65} & $\varepsilon^{\prime}=17.7967$, & $\varepsilon^{\prime}=24.4368$, & $\varepsilon^{\prime}=23.4129$, & $\varepsilon^{\prime}=21.4675$, & $\varepsilon^{\prime}=20.8372$, \\
\cline { 3 - 7 } & & $\varepsilon^{\prime \prime}=10.7801$ & $\varepsilon^{\prime \prime}=11.9121$ & $\varepsilon^{\prime \prime}=12.5610$ & $\varepsilon^{\prime \prime}=1.0781$ & $\varepsilon^{\prime \prime}=0.9662$ \\
\hline \multirow{2}{*}{$\mathrm{MoSe}_{2}$} & \multirow{2}{*}{0.70} & $\varepsilon^{\prime}=21.3204$, & $\varepsilon^{\prime}=20.3560$, & $\varepsilon^{\prime}=19.3366$, & $\varepsilon^{\prime}=17.7994$, & $\varepsilon^{\prime}=14.9028$, \\
\cline { 3 - 7 } & & $\varepsilon^{\prime \prime}=10.9486$ & $\varepsilon^{\prime \prime}=9.3039$ & $\varepsilon^{\prime \prime}=8.4366$ & $\varepsilon^{\prime \prime}=7.0709$ & $\varepsilon^{\prime \prime}=2.7611$, \\
\hline \multirow{2}{*}{$\mathrm{WS}_{2}$} & \multirow{2}{*}{0.80} & $\varepsilon^{\prime}=12.0258$, & $\varepsilon^{\prime}=23.8511$, & $\varepsilon^{\prime}=19.9701$, & $\varepsilon^{\prime}=16.0968$, & $\varepsilon^{\prime}=25.0445$, \\
\cline { 3 - 7 } & & $\varepsilon^{\prime \prime}=4.2578$ & $\varepsilon^{\prime \prime}=3.0578$ & $\varepsilon^{\prime \prime}=1.8420$ & $\varepsilon^{\prime \prime}=0.3203$ & $\varepsilon^{\prime \prime}=2.5676$, \\
\hline \multirow{2}{*}{$\mathrm{WSe}_{2}$} & \multirow{2}{*}{0.70} & $\varepsilon^{\prime}=19.4125$, & $\varepsilon^{\prime}=20.5156$, & $\varepsilon^{\prime}=18.7344$, & $\varepsilon^{\prime}=19.0563$, & $\varepsilon^{\prime}=23.6474$, \\
\cline { 3 - 7 } & & $\varepsilon^{\prime \prime}=8.4135$ & $\varepsilon^{\prime \prime}=3.9423$ & $\varepsilon^{\prime \prime}=2.1955$ & $\varepsilon^{\prime \prime}=0.3205$ & $\varepsilon^{\prime \prime}=2.3801$ \\
\hline
\end{tabular}

Table 3. The optical constants of $2 \mathrm{D} \mathrm{MoS}, \mathrm{MoSe}_{2}, \mathrm{WS}_{2}$, and WSe $\mathrm{With}_{2}$ different excitation wavelengths from $600 \mathrm{~nm}$ to $1024 \mathrm{~nm}$. The dielectric constant is described by $\varepsilon=\varepsilon^{\prime}+\varepsilon^{\prime \prime} i$, where the real part $\varepsilon^{\prime}$ relates to the stored energy within the medium and the imaginary part $\varepsilon^{\prime \prime}$ relates to the dissipation of energy within the medium. (The complex refractive index $\bar{n}$ is defined as $\bar{n}=\sqrt{\varepsilon}=n+\kappa i$, where the real part $n$ indicates the phase velocity, while the imaginary part $\kappa$ known as the extinction coefficient refers to the mass attenuation coefficient).

To obtain the reflected intensity, Fresnel equations and relevant boundary conditions were introduced. In the calculations, the first boundary of the tangential fields was assumed as $Z_{1}=0$, and the tangential fields at the last boundary $Z_{N-1}$, there by giving rise to Eq. (5) as follows:

$$
\left[\begin{array}{l}
U_{1} \\
V_{1}
\end{array}\right]=M\left[\begin{array}{l}
U_{N-1} \\
V_{N-1}
\end{array}\right]
$$

where $U_{1}, U_{N-1}$ represent the tangential components of the first and the last layers in the electric fields, while $V_{1}$, $V_{N-1}$ denote the corresponding components in magnetic fields. $M$ refers to the characteristic matrix of the $N$-layer model. For the $p$-polarized light, the characteristics matrix is given by:

$$
M=\prod_{k=2}^{N-1} M_{k}=\left[\begin{array}{ll}
M_{11} & M_{12} \\
M_{21} & M_{22}
\end{array}\right]
$$

With

$$
M_{k}=\left[\begin{array}{cc}
\cos \beta_{k} & \left(-i \sin \beta_{k}\right) / q_{k} \\
-i q_{k} \sin \beta_{k} & \cos \beta_{k}
\end{array}\right]
$$

Where

$$
\begin{gathered}
q_{k}=\frac{\left(\varepsilon_{k}-n_{1}{ }^{2} \sin ^{2} \theta_{1}\right)^{1 / 2}}{\varepsilon_{k}}=\frac{\lambda \beta_{k}}{2 \pi d_{k} \varepsilon_{k}} \\
\beta_{k}=\frac{2 \pi n_{k} \cos \theta_{k}\left(Z_{k}-Z_{k-1}\right)}{\lambda}
\end{gathered}
$$

Thus, the four elements $M_{11}, M_{12}, M_{21}$ and $M_{22}$ of the matrix $M$ can be calculated. According to the Fresnel's equations, the complex reflection coefficient $r_{p}$ of $p$-polarized incident electromagnetic field can be described by:

$$
r_{p}=\frac{\left(M_{11}+M_{12} q_{N}\right) q_{1}-\left(M_{21}+M_{22} q_{N}\right)}{\left(M_{11}+M_{12} q_{N}\right) q_{1}+\left(M_{21}+M_{22} q_{N}\right)}
$$

where, $q_{1}$ and $q_{N}$ can be calculated from Eq. (8), which represent the relative components of the first layer and the $N_{\text {th }}$ layer respectively. Therefore, the system reflectivity $R_{p}$ for the $p$-polarized incident light can then be obtained by taking the square of the reflection coefficient $r_{p}$, shown as follows:

$$
R_{p}=\left|r_{p}\right|^{2}
$$

The angular sensitivity is defined as the ratio between the change of the resonance angle to the change of the analyte refractive index ${ }^{5}$ in Eq. (12).

$$
S \equiv \frac{d \theta_{S P R}}{d n_{a}}
$$

It can be simplified to 


$$
S=\frac{\Delta \theta_{S P R}}{\Delta n_{a}}
$$

The sensitivity describes the change in the optical signal (i.e., changes in the resonance angle) corresponding to the minute changes in the refractive index of the biomolecular analyte. The sensitivity depends on the evanescent field strength which directly related to the absorbed light energy. Another key parameter of interest is the width of the SPR curve, which related to the accuracy of the sensing system. The width of SPR curve is determined by the dielectric function of the metallic silicon- $\mathrm{MX}_{2}$ thin film. Generally, a large value of $\frac{\left|\varepsilon^{\prime}\right|}{\left|\varepsilon^{\prime \prime}\right|}$. sults in a narrow resonance curve $^{6}$. In this work, the full width at half maximum (FWHM) is calculated to investigate the SPR curve width, as shown in Eq. (14),

$$
F W H M=\frac{1}{2} \times\left(\theta_{\min }+\theta_{\max }\right)
$$

where $\theta_{\min }$ is the incident angle corresponding to the minimum reflected intensity, and $\theta_{\max }$ is the incident angle with the maximum reflected intensity base on the SPR curve.

\section{References}

1. Zeng, S., Baillargeat, D., Ho, H. P. \& Yong, K. T. Nanomaterials enhanced surface plasmon resonance for biological and chemical sensing applications. Chem. Soc. Rev. 43, 3426-3452 (2014).

2. Zeng, S. et al. Graphene-gold metasurface architectures for ultrasensitive plasmonic biosensing. Adv. Mater. 27, 6163-6169 (2015).

3. Homola, J. \& Piliarik, M. Surface Plasmon Resonance Based Sensors. Vol. 4 Ch. 2, 46-47 (Springer, 2006).

4. Zeng, S. et al. A review on functionalized gold nanoparticles for biosensing applications. Plasmonics. 6, 491-506 (2011).

5. Shalabney, A. \& Abdulhalim, I. Sensitivity-enhancement methods for surface plasmon sensors. Laser Photonics Rev. 5, 571-606 (2011).

6. Kooyman, R. P. H. Handbook of Surface Plasmon Resonance. (eds Schasfoort, R. B. \& Tudos, A. J.) Ch. 2, 15-34 (Royal Society of Chemistry, 2008)

7. Kretschmann, E. \& Raether, H. Notizen. Radiative decay of non radiative surface plasmons excited by light. Z. Naturforsch. A. 23, $2135-2136$ (1968).

8. Otto, A. Excitation of nonradiative surface plasma waves in silver by the method of frustrated total reflection. Z. Phys. 216, 398-410 (1968).

9. Kabashin, A. et al. Plasmonic nanorod metamaterials for biosensing. Nat. Mater. 8, 867-871 (2009).

10. Zhu, M. et al. Low-temperature in situ growth of graphene on metallic substrates and its application in anticorrosion. ACS Appl. Mater. Interfaces. 8, 502-510 (2015).

11. Zhu, M. et al. A wafer-scale graphene and ferroelectric multilayer for flexible and fast-switched modulation applications. Nanoscale. 7, 14730-14737 (2015)

12. Wu, L., Chu, H., Koh, W. \& Li, E. Highly sensitive graphene biosensors based on surface plasmon resonance. Opt. Express. 18, 14395-14400 (2010).

13. Zeng, S. et al. Graphene- $\mathrm{MoS}_{2}$ hybrid nanostructures enhanced surface plasmon resonance biosensors. Sens. Actuators B Chem. 207, 801-810 (2015).

14. Mishra, A. K., Mishra, S. K. \& Verma, R. K. Graphene and beyond graphene $\mathrm{MoS}_{2}$ : a new window in surface-plasmon-resonancebased fiber optic sensing. J. Phys. Chem. C. 120, 2893-2900 (2016).

15. Verma, R., Gupta, B. D. \& Jha, R. Sensitivity enhancement of a surface plasmon resonance based biomolecules sensor using graphene and silicon layers. Sens. Actuators B Chem. 160, 623-631 (2011).

16. Homola, J. Surface plasmon resonance sensors for detection of chemical and biological species. Chem. Rev. 108, 462-493 (2008).

17. Zhang, N. et al. Side-channel photonic crystal fiber for surface enhanced Raman scattering sensing. Sens. Actuators B Chem. 223, 195-201 (2016).

18. $\mathrm{Lu}$, X. et al. Large-area synthesis of monolayer and few-layer $\mathrm{MoSe}_{2}$ films on $\mathrm{SiO}_{2}$ substrates. Nano Lett. 14, 2419-2425 (2014).

19. Ross, J. S. et al. Electrical control of neutral and charged excitons in a monolayer semiconductor. Nat. Commun. 4, 1474 (2013).

20. Wang, Q. H., Kalantar-Zadeh, K., Kis, A., Coleman, J. N. \& Strano, M. S. Electronics and optoelectronics of two-dimensional transition metal dichalcogenides. Nat. Nanotechnol. 7, 699-712 (2012).

21. Kuc, A., Zibouche, N. \& Heine, T. Influence of quantum confinement on the electronic structure of the transition metal sulfide $\mathrm{TS}_{2}$. Phys. Rev. B. 83, 245213 (2011).

22. Mak, K. F., Lee, C., Hone, J., Shan, J. \& Heinz, T. F. Atomically thin $\mathrm{MoS}_{2}$ : a new direct-gap semiconductor. Phys. Rev. Lett. 105, 136805 (2010).

23. Radisavljevic, B., Radenovic, A., Brivio, J., Giacometti, V. \& Kis, A. Single-layer $\mathrm{MoS}_{2}$ transistors. Nat. Nanotechnol. 6, 147-150 (2011).

24. Tsai, D. S. et al. Few-layer $\mathrm{MoS}_{2}$ with high broadband photogain and fast optical switching for use in harsh environments. Acs Nano. 7, 3905-3911 (2013).

25. Yu, L. et al. Graphene/MoS hybrid technology for large-scale two-dimensional electronics. Nano Lett. 14, 3055-3063 (2014).

26. Roy, K. et al. Graphene-MoS hybrid structures for multifunctional photoresponsive memory devices. Nat. Nanotechnol. 8, 826-830 (2013).

27. Britnell, L. et al. Strong light-matter interactions in heterostructures of atomically thin films. Science. 340, 1311-1314 (2013)

28. Georgiou, T. et al. Vertical field-effect transistor based on graphene- $\mathrm{WS}_{2}$ heterostructures for flexible and transparent electronics. Nat. Nanotechnol. 8, 100-103 (2013).

29. Cong, C. et al. Synthesis and optical properties of large-area single-crystalline $2 \mathrm{D}$ semiconductor $\mathrm{WS}_{2}$ monolayer from chemical vapor deposition. Adv. Opt. Mater. 2, 131-136 (2014).

30. Shi, H., Pan, H., Zhang, Y. W. \& Yakobson, B. I. Quasiparticle band structures and optical properties of strained monolayer $\mathrm{MoS}_{2}$ and $\mathrm{WS}_{2}$. Phys. Rev. B. 87, 155304 (2013).

31. Eda, G. \& Maier, S. A. Two-dimensional crystals: managing light for optoelectronics. Acs Nano. 7, 5660-5665 (2013).

32. Sygletou, M. et al. Laser induced nucleation of plasmonic nanoparticles on two-dimensional nanosheets for organic photovoltaics. J. Mater. Chem. A. 4, 1020-1027 (2016).

33. Jones, A. M. et al. Optical generation of excitonic valley coherence in monolayer WSe ${ }_{2}$. Nat. Nanotechnol. 8, 634-638 (2013).

34. Seyler, K. L. et al. Electrical control of second-harmonic generation in a $\mathrm{WSe}_{2}$ monolayer transistor. Nat. Nanotechnol. 10, 407-411 (2015).

35. Koperski, M. et al. Single photon emitters in exfoliated WSe ${ }_{2}$ structures. Nat. Nanotechnol. 10, 503-506 (2015). 
36. Ross, J. S. et al. Electrically tunable excitonic light-emitting diodes based on monolayer WSe $\mathrm{W}_{2}$ pn junctions. Nat. Nanotechnol. 9, 268-272 (2014)

37. Tongay, S. et al. Thermally driven crossover from indirect toward direct bandgap in 2D semiconductors: $\mathrm{MoSe}_{2}$ versus $\mathrm{MoS}_{2}$. Nano Lett. 12, 5576-5580 (2012).

38. Fan, C. et al. Low temperature electrical and photo-responsive properties of $\mathrm{MoSe}_{2}$. Appl. Phys. Lett. 104, 202105 (2014).

39. Huang, C. et al. Lateral heterojunctions within monolayer $\mathrm{MoSe}_{2}-\mathrm{WSe}_{2}$ semiconductors. Nat. Mater. 13, 1096-1101 (2014).

40. Tonndorf, P. et al. Photoluminescence emission and Raman response of monolayer $\mathrm{MoS}_{2}, \mathrm{MoSe}_{2}$, and $\mathrm{WSe}_{2}$. Opt. Express. 21, 4908-4916 (2013).

41. Lee, S. H. et al. High-performance photocurrent generation from two-dimensional WS $\mathrm{W}_{2}$ field-effect transistors. Appl. Phys. Lett. 104, 193113 (2014).

42. Hoggard, A. et al. Using the plasmon linewidth to calculate the time and efficiency of electron transfer between gold nanorods and graphene. Acs Nano. 7, 11209 (2013).

43. Fang, Z. et al. Gated tunability and hybridization of localized plasmons in nanostructured graphene. Acs Nano. 7, 2388 (2013).

44. Giovannetti, G. et al. Doping graphene with metal contacts. Phys. Rev. Lett. 101, 026803 (2008).

45. Lee, J. et al. Two-dimensional layered $\mathrm{MoS}_{2}$ biosensors enable highly sensitive detection of biomolecules. Sci. Rep. 4, (2014).

46. Zhu, C. et al. Single-layer $\mathrm{MoS}_{2}$-based nanoprobes for homogeneous detection of biomolecules. J. Am. Chem. Soc. 135, 5998-6001 (2013).

47. Homola, J., Yee, S. S. \& Gauglitz, G. Surface plasmon resonance sensors: review. Sens. Actuators B Chem. 54, 3-15 (1999).

48. Lahav, A., Auslender, M. \& Abdulhalim, I. Sensitivity enhancement of guided-wave surface-plasmon resonance sensors. Opt. Lett. 33, 2539-2541 (2008).

49. Shalabney, A. \& Abdulhalim, I. Electromagnetic fields distribution in multilayer thin film structures and the origin of sensitivity enhancement in surface plasmon resonance sensors. Sens. Actuators A Phys. 159, 24-32 (2010).

50. Polyanskiy, M. N. Refractive index database. Available at: http://refractiveindex.info.

51. Johnson, P. B. \& Christy, R. W. Optical constants of the noble metals. Phys. Rev. B. 6, 4370 (1972)

52. Raether, H. Surface Plasmons on Smooth Surfaces. Vol. 111, Ch. 2, 4-39 (Springer, 1988).

53. Gupta, B. \& Sharma, A. K. Sensitivity evaluation of a multi-layered surface plasmon resonance-based fiber optic sensor: a theoretical study. Sens. Actuators B Chem. 107, 40-46 (2005).

54. Auslender, M. \& Hava, S. Handbook of Optical Constants of Solids, Vol. 3 (eds Palik, E. D.) Ch. 6, 155-186 (Elsevier, 1998).

55. Li, Y. et al. Measurement of the optical dielectric function of monolayer transition-metal dichalcogenides: $\mathrm{MoS}_{2}, \mathrm{MoSe}_{2}, \mathrm{WS}_{2}$, and $\mathrm{WSe}_{2}$. Phys. Rev. B. 90, 205422 (2014).

56. Liu, H. L. et al. Optical properties of monolayer transition metal dichalcogenides probed by spectroscopic ellipsometry. Appl. Phys. Lett. 105, 201905 (2014).

\section{Acknowledgements}

This work was supported by the Singapore Ministry of Education (Grants Tier 2 MOE2010-T2-2-010 (M4020020.040 ARC2/11) and Tier 1 M4010360.040 RG29/10), NTU-NHG Innovation Collaboration Grant (No. M4061202.040), A*STAR Science and Engineering Research Council (No. M4070176.040), School of Electrical and Electronic Engineering at NTU, Guangdong Natural Science Foundation (2014A030312008) and Basic Research Foundation of Shenzhen (JCYJ20140418095735543).

\section{Author Contributions}

Q.O., S.Z. and K.-T.Y. conceived the idea. Q.O., S.Z., L.J., L.H., G.X. and J.Q. designed and carried out the research. Q.O., S.Z. and K.-T.Y. co-wrote the manuscript. X.-Q.D., J.Q., S.H. and P.C. provided suggestions, and all authors commented on the manuscript.

\section{Additional Information}

Supplementary information accompanies this paper at http://www.nature.com/srep

Competing financial interests: The authors declare no competing financial interests.

How to cite this article: Ouyang, Q. et al. Sensitivity Enhancement of Transition Metal Dichalcogenides/Silicon Nanostructure-based Surface Plasmon Resonance Biosensor. Sci. Rep. 6, 28190; doi: 10.1038/srep28190 (2016).

(c) (i) This work is licensed under a Creative Commons Attribution 4.0 International License. The images or other third party material in this article are included in the article's Creative Commons license, unless indicated otherwise in the credit line; if the material is not included under the Creative Commons license, users will need to obtain permission from the license holder to reproduce the material. To view a copy of this license, visit http://creativecommons.org/licenses/by/4.0/ 